\title{
HEIGHT AND WEIGHT NORMS AND SOMATOTYPIC HEIGHT-WEIGHT CLASSIFICATION OF ESTONIAN SCHOOLCHILDREN AGED 7-18 YEARS
}

\author{
Helje KaArma ${ }^{1}$, Gudrun Veldre ${ }^{1}$, Liidia Saluste ${ }^{1}$, \\ Mart Lintsi ${ }^{1}$, JaAn Kasmel ${ }^{1}$, Ene-Margit Titt ${ }^{2}$, Raini Stamm ${ }^{1}$, \\ Maie Toomsalu ${ }^{1}$, Erik Salm ${ }^{2}$, Ene Kä̈̈rik ${ }^{2}$, Andres Arend ${ }^{3}$ \\ ${ }^{1}$ Centre for Physical Anthropology, Department of Anatomy, \\ University of Tartu, Tartu, Estonia \\ ${ }^{2}$ Institute of Mathematical Statistics, University of Tartu, Tartu, Estonia \\ ${ }^{3}$ Department of Anatomy, University of Tartu, Tartu, Estonia
}

\begin{abstract}
Relying on regularities of Estonian schoolchildren's body build, height and weight norms and a somatotypic height-weight classification were created for Estonian schoolchildren aged $7-18$ years.
\end{abstract}

Keywords: body build; height-weight classification; anthropometry

\section{INTRODUCTION}

In Issue 1 of our journal Papers on Anthropology of the previous year (2017), we presented the mean values of height and weight of adult Estonian population (aged 20-70 years) as national norms and somatotypic classifications for men and women of each year of age [1].

\section{METHODS}

In the current study, we planned to do the same on the sample of boys and girls aged 7-18 years. The study is based on the following samples:

1) South Estonian schoolgirls aged $7-18$ years $(n=1500)$ [2].

2) Girls of secondary schools of Tartu aged $15-18$ years $(n=1114)$ [5].

3) Conscripts - young men aged $17-18$ years $(n=1500)$ [3]. 
4) The latest data on schoolchildren's height and weight from Estonian schools, 2006-2009 ( $\mathrm{n}=21,764 ; 10,282$ boys, 11,482 girls) [4].

\section{RESULTS}

Based on the first three samples, we found that, if the sample of subjects is studied according to years of age and all the anthropometric variables measured are classified in a 5 SD height-weight classification (see Figure 1), body build regularities similar to adults were also revealed in schoolchildren.

The height and weight data of schoolgirls and -boys aged 7-18 years of the last sample $(n=21,764)$ are presented as the average national norms (see Tables 1 and 2).

The data of all subjects were placed into height-weight classes according to ages 7-18 in absolute numbers and percentages (see Tables $3,4,5,6$ ). The division into classes was made somewhat more detailed, and the classes of pycnomorphs and leptomorphs were respectively divided into three subclasses (see Figure 2).

Finally, the limit values of classes are presented for all boys and girls for each year of age (7-18) (see Tables 7 and 8).

These data can be used for somatotypic assessment of children and for relating body build data to medical, health promotional and nutritional data.

\begin{tabular}{|c|c|c|c|c|}
\hline \multicolumn{5}{|c|}{ Weight classes } \\
\hline \multirow{4}{*}{$\begin{array}{l}\text { Height } \\
\text { classes }\end{array}$} & & Light & Medium & Heavy \\
\hline & Short & Small & & \multirow{2}{*}{$\begin{array}{l}\text { Pycno- } \\
\text { morphic }\end{array}$} \\
\hline & Medium & \multirow{2}{*}{$\begin{array}{l}\text { Lepto- } \\
\text { morphic }\end{array}$} & Medium & \\
\hline & Tall & & & Large \\
\hline
\end{tabular}

Figure 1. Body build classes

\begin{tabular}{|c|c|c|c|c|}
\cline { 3 - 5 } \multicolumn{2}{c|}{} & \multicolumn{4}{c|}{ Weight classes } \\
\cline { 2 - 5 } \multicolumn{2}{c|}{} & Light & Medium & Heavy \\
\hline \multirow{3}{*}{$\begin{array}{l}\text { Height } \\
\text { classes }\end{array}$} & Short & 1.Small & 5. Pycnomorphic & II \\
\cline { 2 - 5 } & Medium & 4. Leptomophic I & 2. Medium & III \\
\cline { 2 - 5 } & Tall & II & III & 3. Large \\
\hline
\end{tabular}

Figure 2. Body build classes 
Table 1. Mean values of height and weight for Estonian schoolboys aged 7-18 years $(n=10062)$.

\begin{tabular}{cccccccccc}
\hline \multirow{2}{*}{ Age } & \multirow{2}{*}{$\mathbf{N}$} & \multicolumn{9}{c}{ Height } & Min & Max & Mean & SD & Min & Max & Mean & SD \\
\hline 7 & 936 & 111.50 & 150.00 & 129.61 & 5.71 & 13.30 & 49.00 & 28.30 & 5.16 \\
8 & 705 & 114.00 & 155.50 & 133.73 & 5.94 & 19.50 & 55.50 & 30.51 & 5.93 \\
9 & 1052 & 119.00 & 162.00 & 139.85 & 6.23 & 19.00 & 63.00 & 34.70 & 7.19 \\
10 & 456 & 126.00 & 165.00 & 144.60 & 6.38 & 24.90 & 65.00 & 38.76 & 8.63 \\
11 & 1114 & 129.90 & 175.60 & 151.35 & 7.10 & 26.00 & 84.00 & 43.24 & 9.74 \\
12 & 569 & 134.00 & 183.50 & 156.23 & 7.98 & 25.11 & 84.00 & 46.91 & 10.36 \\
13 & 1227 & 140.00 & 193.00 & 165.51 & 8.73 & 28.00 & 95.50 & 55.06 & 11.81 \\
14 & 680 & 142.70 & 195.00 & 169.62 & 8.65 & 29.70 & 98.50 & 58.59 & 11.56 \\
15 & 1596 & 151.50 & 200.00 & 176.75 & 7.34 & 35.80 & 103.00 & 65.84 & 10.94 \\
16 & 831 & 154.50 & 200.00 & 178.55 & 7.10 & 40.00 & 104.00 & 67.59 & 10.59 \\
17 & 373 & 162.00 & 202.00 & 180.82 & 6.24 & 47.00 & 107.00 & 71.50 & 11.10 \\
18 & 523 & 160.00 & 200.10 & 181.28 & 6.53 & 47.00 & 110.00 & 74.12 & 11.06 \\
\hline
\end{tabular}

Table 2. Mean values of height and weight for Estonian schoolgirls aged 7-18 years $(n=11204)$.

\begin{tabular}{cccccccccc}
\hline \multirow{2}{*}{ Age } & \multirow{2}{*}{$\mathbf{N}$} & \multicolumn{4}{c}{ Height } & \multicolumn{5}{c}{ Weight } \\
\cline { 3 - 9 } & & Min & Max & Mean & SD & Min & Max & Mean & SD \\
\hline 7 & 1041 & 110.50 & 146.50 & 128.24 & 5.60 & 15.80 & 44.50 & 27.05 & 4.85 \\
8 & 667 & 115.30 & 148.80 & 132.89 & 5.92 & 18.00 & 47.40 & 29.73 & 5.65 \\
9 & 1063 & 121.00 & 162.00 & 139.45 & 6.44 & 16.50 & 57.00 & 33.83 & 6.75 \\
10 & 450 & 120.50 & 172.00 & 145.13 & 7.47 & 20.40 & 69.00 & 37.88 & 8.89 \\
11 & 1298 & 128.50 & 175.00 & 152.16 & 7.28 & 21.00 & 75.50 & 43.13 & 9.28 \\
12 & 536 & 134.00 & 173.00 & 157.70 & 6.90 & 26.00 & 80.70 & 47.77 & 9.76 \\
13 & 1355 & 141.00 & 183.50 & 163.39 & 6.43 & 30.00 & 87.00 & 53.26 & 9.56 \\
14 & 637 & 143.50 & 185.00 & 165.20 & 6.54 & 33.20 & 87.20 & 55.38 & 10.01 \\
15 & 1829 & 149.30 & 188.00 & 167.13 & 5.95 & 32.70 & 91.20 & 58.44 & 8.90 \\
16 & 978 & 149.50 & 189.00 & 167.63 & 6.24 & 35.50 & 88.50 & 58.87 & 8.35 \\
17 & 588 & 150.00 & 188.00 & 168.62 & 5.88 & 43.00 & 91.50 & 60.84 & 8.57 \\
18 & 762 & 149.00 & 184.00 & 168.23 & 5.88 & 41.00 & 93.00 & 60.76 & 9.15 \\
\hline
\end{tabular}


Table 3. The distribution of Estonian schoolboys in height-weight classes according to years of age ( $7-18$ years)

\begin{tabular}{ccccccccccc}
\hline \multirow{2}{*}{ Age } & \multirow{2}{*}{ Small } & \multirow{2}{*}{ Medium } & Large & \multicolumn{3}{c}{ Pycnomorphs } & \multicolumn{3}{c}{ Leptomorphs } & \multirow{2}{*}{ Total } \\
\cline { 5 - 8 } $\mathbf{7}$ & 222 & 188 & 171 & 59 & 13 & 57 & 102 & 10 & 114 & 936 \\
$\mathbf{8}$ & 161 & 156 & 113 & 41 & 8 & 44 & 79 & 11 & 92 & 705 \\
$\mathbf{9}$ & 240 & 196 & 177 & 71 & 10 & 67 & 128 & 23 & 140 & 1052 \\
10 & 102 & 85 & 73 & 35 & 9 & 24 & 55 & 10 & 63 & 456 \\
11 & 247 & 230 & 171 & 84 & 17 & 66 & 122 & 20 & 157 & 1114 \\
12 & 127 & 98 & 104 & 44 & 16 & 44 & 79 & 5 & 52 & 569 \\
13 & 303 & 206 & 233 & 94 & 20 & 85 & 116 & 4 & 166 & 1227 \\
14 & 155 & 122 & 117 & 36 & 16 & 65 & 67 & 6 & 96 & 680 \\
15 & 312 & 294 & 264 & 146 & 39 & 128 & 163 & 38 & 212 & 1596 \\
16 & 152 & 161 & 123 & 70 & 22 & 89 & 89 & 20 & 105 & 831 \\
17 & 64 & 75 & 52 & 33 & 5 & 34 & 44 & 12 & 54 & 373 \\
18 & 102 & 98 & 68 & 52 & 19 & 48 & 52 & 21 & 63 & 523 \\
\hline Total & 2187 & 1909 & 1666 & 765 & 194 & 751 & 1096 & 180 & 1314 & 10062 \\
\hline
\end{tabular}

Table 4. The percentage distribution of Estonian schoolboys in height-weight classes by years of age ( $7-18$ years)

\begin{tabular}{cccccccccccc}
\hline \multirow{2}{*}{ Age } & \multirow{2}{*}{ Small } & \multirow{2}{*}{ Medium } & Large & \multicolumn{3}{c}{ Pycnomorphs } & \multicolumn{3}{c}{ Leptomorphs } & \multirow{2}{*}{ Total } \\
\cline { 6 - 9 } & & & & I & II & III & I & II & III & \\
\hline $\mathbf{7}$ & 23.72 & 20.09 & 18.27 & 6.30 & 1.39 & 6.09 & 10.90 & 1.07 & 12.18 & 100.00 \\
$\mathbf{8}$ & 22.84 & 22.13 & 16.03 & 5.82 & 1.13 & 6.24 & 11.21 & 1.56 & 13.05 & 100.00 \\
$\mathbf{9}$ & 22.81 & 18.63 & 16.83 & 6.75 & 0.95 & 6.37 & 12.17 & 2.19 & 13.31 & 100.00 \\
10 & 22.37 & 18.64 & 16.01 & 7.68 & 1.97 & 5.26 & 12.06 & 2.19 & 13.82 & 100.00 \\
11 & 22.17 & 20.65 & 15.35 & 7.54 & 1.53 & 5.92 & 10.95 & 1.80 & 14.09 & 100.00 \\
12 & 22.32 & 17.22 & 18.28 & 7.73 & 2.81 & 7.73 & 13.88 & 0.88 & 9.14 & 100.00 \\
13 & 24.69 & 16.79 & 18.99 & 7.66 & 1.63 & 6.93 & 9.45 & 0.33 & 13.53 & 100.00 \\
14 & 22.79 & 17.94 & 17.21 & 5.29 & 2.35 & 9.56 & 9.85 & 0.88 & 14.12 & 100.00 \\
15 & 19.55 & 18.42 & 16.54 & 9.15 & 2.44 & 8.02 & 10.21 & 2.38 & 13.28 & 100.00 \\
16 & 18.29 & 19.37 & 14.80 & 8.42 & 2.65 & 10.71 & 10.71 & 2.41 & 12.64 & 100.00 \\
17 & 17.16 & 20.11 & 13.94 & 8.85 & 1.34 & 9.12 & 11.80 & 3.22 & 14.48 & 100.00 \\
18 & 19.50 & 18.74 & 13.00 & 9.94 & 3.63 & 9.18 & 9.94 & 4.02 & 12.05 & 100.00 \\
\hline Total & 21.74 & 18.97 & 16.56 & 7.60 & 1.93 & 7.46 & 10.89 & 1.79 & 13.06 & 100.00 \\
\hline
\end{tabular}


Table 5. The distribution of Estonian schoolgirls in height-weight classes by years of age (7-18 years)

\begin{tabular}{ccccccccccc}
\hline \multirow{2}{*}{ Age } & \multirow{2}{*}{ Small } & Medium & Large & \multicolumn{3}{c}{ Pycnomorphs } & \multicolumn{3}{c}{ Leptomorphs } & \multirow{2}{*}{ Total } \\
\cline { 7 - 9 } & & & & I & II & III & I & II & III & \\
\hline $\mathbf{7}$ & 232 & 225 & 171 & 107 & 13 & 121 & 73 & 13 & 86 & 1041 \\
$\mathbf{8}$ & 132 & 139 & 111 & 84 & 10 & 82 & 45 & 8 & 56 & 667 \\
$\mathbf{9}$ & 245 & 198 & 191 & 137 & 18 & 106 & 74 & 13 & 81 & 1063 \\
10 & 112 & 86 & 70 & 39 & 7 & 62 & 32 & 4 & 38 & 450 \\
11 & 295 & 210 & 226 & 147 & 11 & 179 & 107 & 19 & 104 & 1298 \\
12 & 126 & 99 & 100 & 45 & 6 & 67 & 42 & 7 & 44 & 536 \\
13 & 271 & 231 & 207 & 135 & 43 & 191 & 118 & 45 & 114 & 1355 \\
14 & 128 & 99 & 97 & 75 & 20 & 86 & 46 & 19 & 67 & 637 \\
15 & 311 & 296 & 248 & 211 & 76 & 206 & 198 & 68 & 215 & 1829 \\
16 & 180 & 156 & 138 & 111 & 36 & 139 & 96 & 31 & 91 & 978 \\
17 & 96 & 92 & 77 & 80 & 24 & 87 & 55 & 17 & 60 & 588 \\
$\mathbf{1 8}$ & 145 & 122 & 115 & 97 & 25 & 87 & 76 & 24 & 71 & 762 \\
\hline Total & 2273 & 1953 & 1751 & 1268 & 289 & 1413 & 962 & 268 & 1027 & 11204 \\
\hline
\end{tabular}

Table 6. The percentage distribution of Estonian schoolgirls in height-weight classes by years of age ( $7-18$ years)

\begin{tabular}{cccccccccccc}
\hline \multirow{2}{*}{ Age } & \multirow{2}{*}{ Small } & \multirow{2}{*}{ Medium } & Large & \multicolumn{9}{c}{ Pycnomorphs } & \multicolumn{3}{c}{ Leptomorphs } & \multirow{2}{*}{ Total } \\
\cline { 6 - 9 } & & & & I & II & III & I & II & III & \\
\hline $\mathbf{7}$ & 22.29 & 21.61 & 16.43 & 10.28 & 1.25 & 11.62 & 7.01 & 1.25 & 8.26 & 100.00 \\
$\mathbf{8}$ & 19.79 & 20.84 & 16.64 & 12.59 & 1.50 & 12.29 & 6.75 & 1.20 & 8.40 & 100.00 \\
$\mathbf{9}$ & 23.05 & 18.63 & 17.97 & 12.89 & 1.69 & 9.97 & 6.96 & 1.22 & 7.62 & 100.00 \\
$\mathbf{1 0}$ & 24.89 & 19.11 & 15.56 & 8.67 & 1.56 & 13.78 & 7.11 & 0.89 & 8.44 & 100.00 \\
$\mathbf{1 1}$ & 22.73 & 16.18 & 17.41 & 11.33 & 0.85 & 13.79 & 8.24 & 1.46 & 8.01 & 100.00 \\
$\mathbf{1 2}$ & 23.51 & 18.47 & 18.66 & 8.40 & 1.12 & 12.50 & 7.84 & 1.31 & 8.21 & 100.00 \\
$\mathbf{1 3}$ & 20.00 & 17.05 & 15.28 & 9.96 & 3.17 & 14.10 & 8.71 & 3.32 & 8.41 & 100.00 \\
$\mathbf{1 4}$ & 20.09 & 15.54 & 15.23 & 11.77 & 3.14 & 13.50 & 7.22 & 2.98 & 10.52 & 100.00 \\
$\mathbf{1 5}$ & 17.00 & 16.18 & 13.56 & 11.54 & 4.16 & 11.26 & 10.83 & 3.72 & 11.76 & 100.00 \\
$\mathbf{1 6}$ & 18.40 & 15.95 & 14.11 & 11.35 & 3.68 & 14.21 & 9.82 & 3.17 & 9.30 & 100.00 \\
$\mathbf{1 7}$ & 16.33 & 15.65 & 13.10 & 13.61 & 4.08 & 14.80 & 9.35 & 2.89 & 10.20 & 100.00 \\
$\mathbf{1 8}$ & 19.03 & 16.01 & 15.09 & 12.73 & 3.28 & 11.42 & 9.97 & 3.15 & 9.32 & 100.00 \\
\hline Total & 20.29 & 17.43 & 15.63 & 11.32 & 2.58 & 12.61 & 8.59 & 2.39 & 9.17 & 100.00 \\
\hline
\end{tabular}




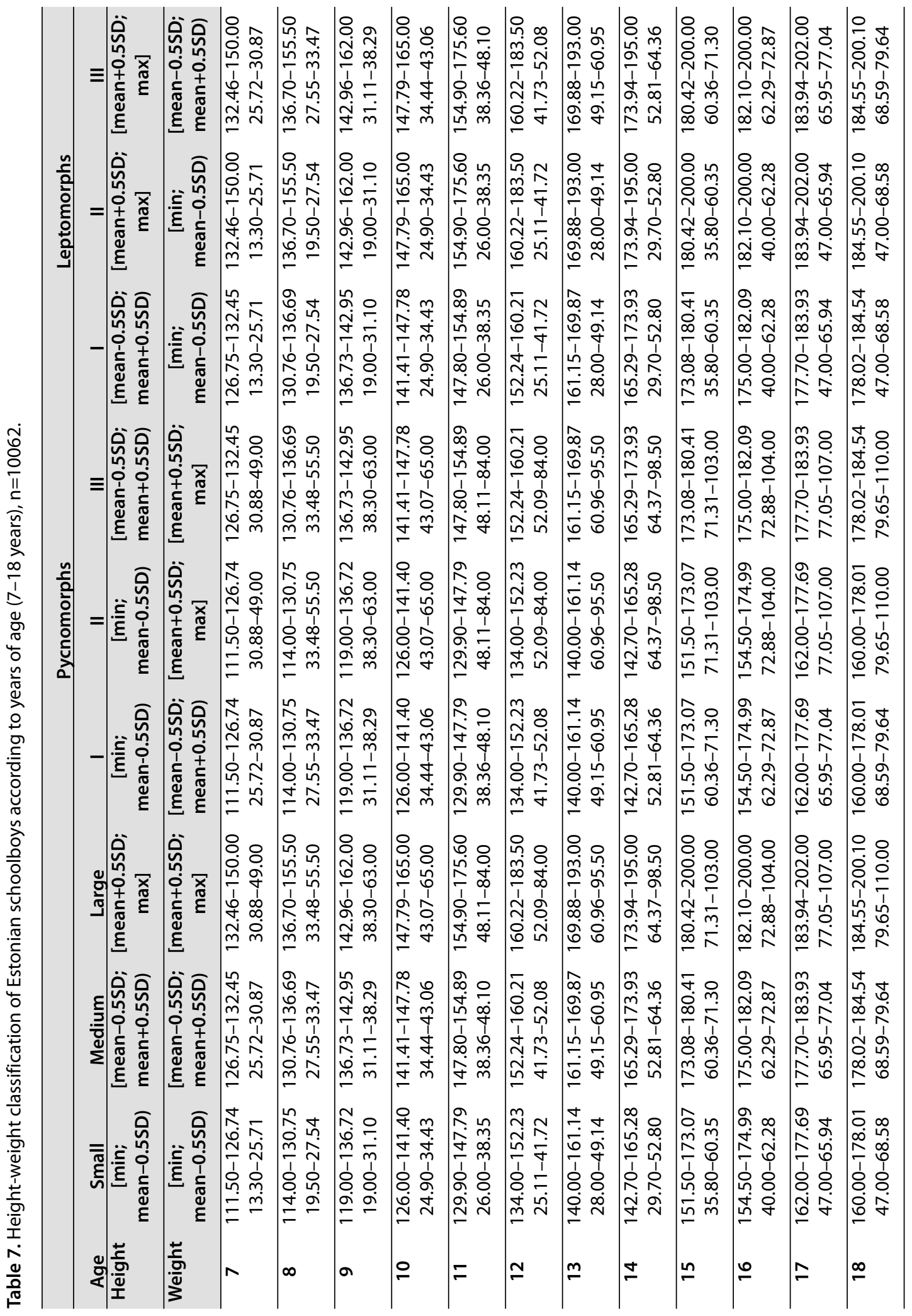




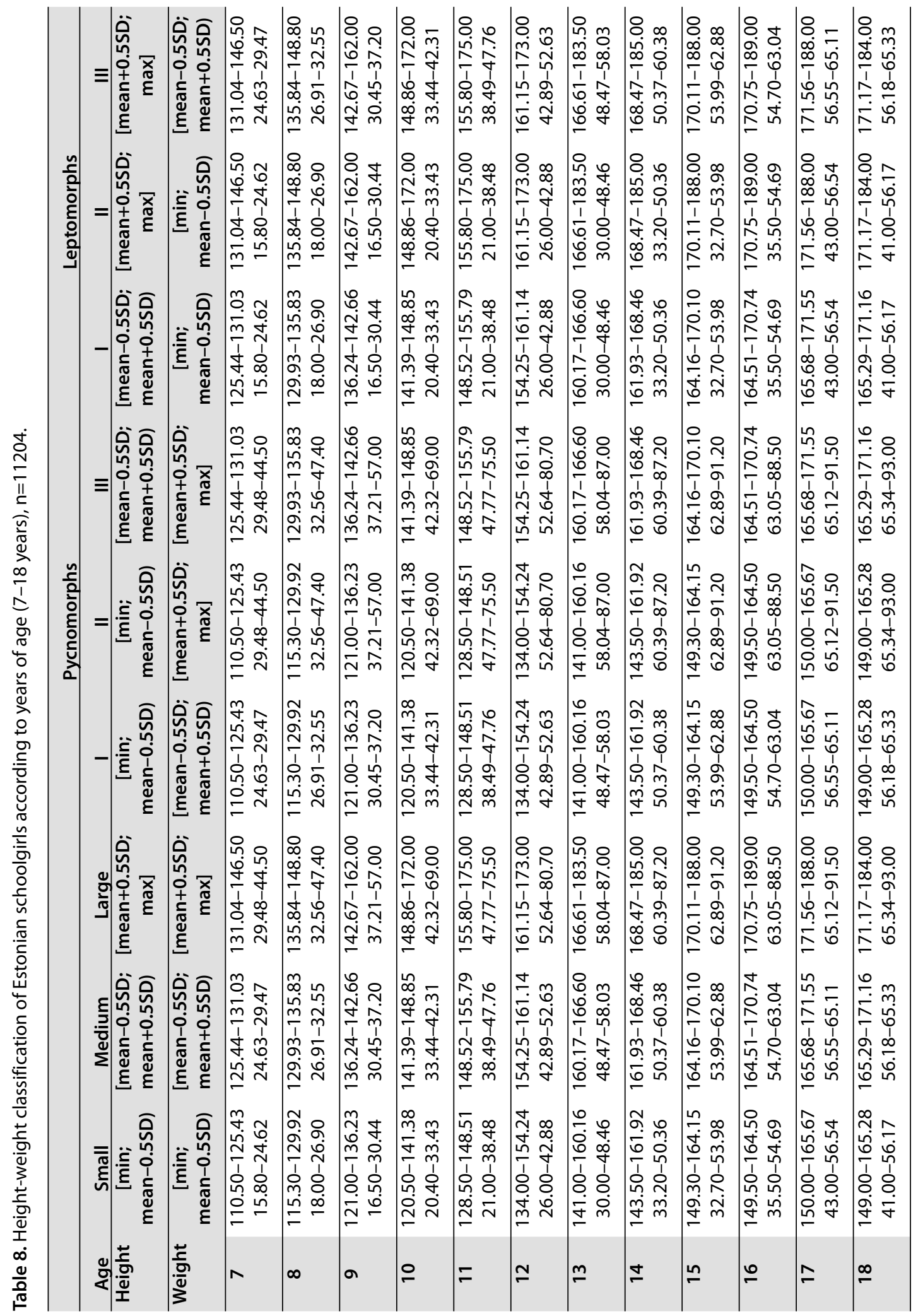




\section{REFERENCES}

1. Kaarma H., Veldre G., Saluste L., Lintsi M., Kasmel J., Tiit E.-M., Stamm R., Toomsalu M., Arend A. (2017) On systematisation of Estonians' body build data. Papers on Anthropology XXVI/1, 9-27.

2. Kaarma H., Stamm R., Kasmel J., Koskel S. (2005) Body build classification for ordinary schoolgirls (aged 7-18 years) and volleyball girls (aged 13-16 years). Anthrop. Anz., 63 (1), 77-92.

3. Lintsi M., Kaarma H. (2013) Five-class height-weight model for systematization of seventeen-year-old recruits' anthropometric data. Antrhop. Anz. 61 (4), 435-443.

4. Salm E., Käärik E., Kaarma H. (2013) The growth charts of Estonian schoolchildren. Comparative analysis. Papers on Anthropology, 2013, 171-183.

5. Veldre G., Stamm R., Koskel S. (2002) A possibility of systematization of anthropological data of girls aged 12-15. Anthrop. Anz. 60 (4), 369-382. 